\title{
DDPG-Based Continuous Thickness and Tension Coupling Control for the Unsteady Cold Rolling Process
}

\section{Wenying Zeng}

Northeastern University https://orcid.org/0000-0003-2866-9622

Jinkuan Wang ( $\nabla$ wjk@neuq.edu.cn )

Northeastern University

Yan Zhang

Northeastern University at Qinhuangdao

Yinghua Han

Northeastern University at Qinhuangdao

Qiang Zhao

Northeastern University at Qinhuangdao

\section{Research Article}

Keywords: Deep reinforcement learning, Cold rolling, Neural network, Thickness and tension control, Unsteady rolling process

Posted Date: December 15th, 2021

DOI: https://doi.org/10.21203/rs.3.rs-1149327/v1

License: (c) (i) This work is licensed under a Creative Commons Attribution 4.0 International License. Read Full License

Version of Record: A version of this preprint was published at The International Journal of Advanced Manufacturing Technology on April 25th, 2022. See the published version at https://doi.org/10.1007/s00170-022-09239-4. 


\title{
DDPG-based continuous thickness and tension coupling
}

\section{control for the unsteady cold rolling process}

\author{
Wenying Zeng ${ }^{1}$, Jinkuan Wang ${ }^{1 \star}$, Yan Zhang ${ }^{2}$, Yinghua Han ${ }^{2}$, Qiang Zhao ${ }^{2}$ \\ ${ }^{1}$ College of Information Science and Engineering, Northeastern University, Shenyang 110819, China. \\ ${ }^{2}$ Engineering Optimization and Smart Antenna Institute, Northeastern University at Qinhuangdao, \\ Qinhuangdao 066004, China.
}

*Jinkuan Wang. E-mail: wjk@ @euq.edu.cn;

\begin{abstract}
s
Cold rolling is an important part of the iron and steel industry, and the unsteady rolling process of cold rolling usually brings significant influences on the stability of product quality. In the unsteady rolling process, various disturbances and uncertainties such as variable lubrication state, variable equipment working conditions lead to difficulties in the establishment of state space model of thickness and tension, which has become a thorny problem in thickness and tension control. In this paper, we present a model-free controller based on Deep Deterministic Policy Gradient(DDPG), which can continuously control the thickness tension of the unsteady rolling process without the mathematical model. We first formulate the thickness and tension control problem to Markov Decision Process(MDP). We apply strategies such as dividing state space variables with mechanism model, defining reward function and state normalization, the random disturbance and complex uncertainties of unsteady cold rolling process are coped with by utilizing the DDPG controller. In addition, these strategies also ensure the learning performance and stability of the DDPG controller under random disturbance. Simulations and experiments show that the proposed the DDPG controller does not require any prior knowledge of uncertain parameters and can operate without knowing unsteady rolling mathematical models, which has better accuracy, stability and rapidity for thickness and tension in the unsteady rolling process than proportional integral(PI) controller. The artificial intelligence-based controller brings both product quality improvement and intelligence to cold rolling.
\end{abstract}

Keywords Deep reinforcement learning, Cold rolling, Neural network, Thickness and tension control, Unsteady rolling process

\section{Introduction}

Cold rolling strip is one of the important parts of strip products, which has been widely used in the automotive industry, aerospace, shipbuilding, bridge, architecture, electronic and household appliance industries. The cold rolling strip is produced by the cold rolling production line composed of stands in which the hot strip passes through each stand in turn, and each stand applies rolling force to the strip through pairs of work rolls, the thickness of the strip reduces continuously. Fig. 1 is the equipment distribution diagram of a five-stand cold rolling line.

The thickness of the strip is important quality indicators of cold rolling strip. In recent years, the demand for the cold rolling strip is constantly increasing, which brings new challenges to the production quality and the production efficiency of the cold rolling strip. Once the tension of the strip is out of range will cause the strip fracture which shows the tension control of the strip is the key to guaranteeing the stable production [1]. For the cold rolling process, there is a strong interaction between strip thickness and tension. Generally, the control strategy of the thickness control and the 
tension is that roll gap controls tension and roll speed adjusts thickness.

The unsteady rolling process is a special part of the cold rolling process which includes acceleration, deceleration and over weld process, and so on. With the advancement of the industrialization process, the control performance of the steady-state rolling process has reached a very high level for the cold rolling process. However, in the unsteady rolling process, various disturbances and uncertainties such as variable lubrication state, variable equipment working conditions lead to difficulties in the establishment of the mathematical model, which cause the thickness and tension control performance in the unsteady rolling process are still inadequate. In addition, the coupling of thickness and tension in the unsteady rolling process also affects the quality of the finished strip.

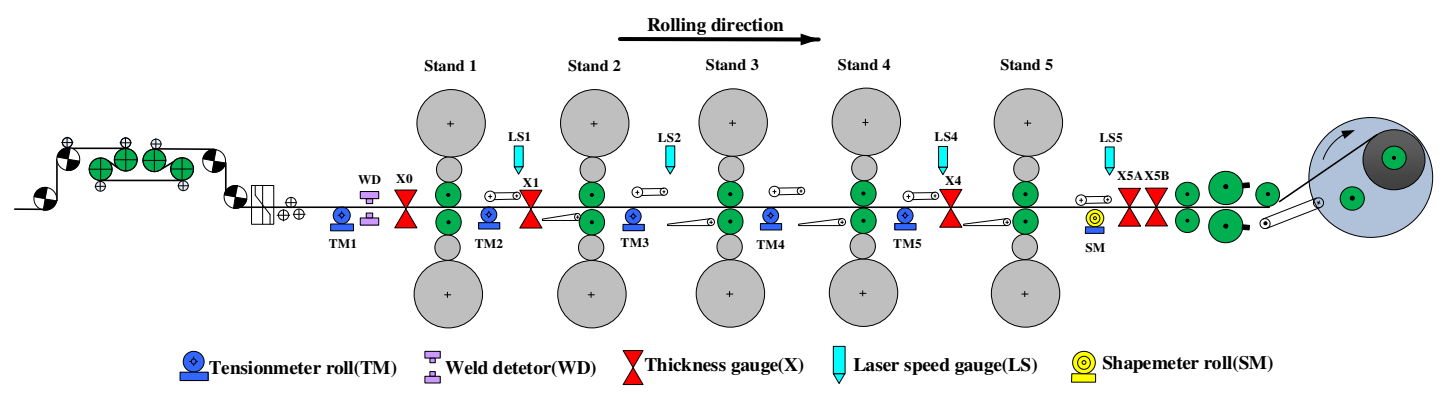

Fig. 1 Equipment distribution of five-stand cold rolling production line

The widely used Automatic Gauge Control(AGC) and Automatic Tension Control(ATC) in the cold rolling process based on PI controller has reached a high level. PI controller has achieved excellent control performance in Single Input Single Output(SISO) control system, but it also has many shortcomings such as inability to solve multivariable coupling problems, lack of flexibility, and poor adaptability. In the actual production process of cold rolling strip, there is a strong correlation and interaction between thickness control system and tension control system.

To better adapt to the actual production process and improve the production quality, many researchers try to use different control theories to control the thickness and tension of cold rolling strips [2]. Wang et al. constructed the mathematical model which considered random actuator failure and designed the fault-tolerant controller for the AGC system to control the thickness of the cold rolling process [3]. Friedel et al. [4] established the rolling process model of a single stand and applied predictive functional control to thickness tension control to improve the accuracy of thickness and tension control. Some researchers focus on decoupling control of thickness and tension. Li et al. [5] designed a decoupling controller based on PID algorithm with the help of a diagonal recurrent neural network and achieved certain results on the proposed dynamic coupling model. In view of the coupling relationship of the thickness and tension, a decoupling compensator based on invariance principle was designed and improve the accuracy of thickness and tension [6]. An [7] designed the multivariable decoupling control system of thickness and tension of cold rolling based on the neural network with the genetic algorithm which can resist small disturbances in the system. However, the above methods did not consider the random disturbance in the unsteady rolling process, which led to the insufficient thickness tension control in the unsteady rolling process.

To improve control performance and enhance system stability, many researchers have established mathematical models considering disturbance terms and applied them to the thickness tension control. $\mathrm{Hu}$ et al. introduced a new state space model of the cold rolling process, proposed the multivariable optimization strategy based on inverse linear quadratic form and the control method based on receding horizon control strategy respectively to optimize the control performance of thickness and tension $[8,9]$. Koofigar et al. [10 established a mathematical model considering the uncertainty of cold rolling process, and used the model to design a robust controller based on $\mathrm{H} \infty$ control theory. Ogasahara et al. [11] designed a controller based on explicit model predictive control, which obtained good control performance in acceleration process which has a small disturbance. In the unsteady rolling process with random disturbance, the performance of the controller is still insufficient. Hu et al. [12] established a state space model with constraints and proposed the distributed model predictive control online optimal control strategy which has good tracking performance compared with PI controller. Ozaki et al. [13] proposed a nonlinear mathematical model to accurately control the tension and thickness of the cold rolling system during acceleration process and deceleration process. In order to analyze the characteristics of the unsteady rolling process, Cao et al. [14] proposed an unsteady lubrication model and developed a dynamic chatter model, and analyzed the effects of the main process parameters on the mill stability. The methods mentioned above can be categorized as model-based methods, and the 
parameters are constant in the unsteady rolling process. However, accurate model parameters cannot be obtained because of many unexpected situations, such as insufficient data samples [15]. In addition, when the system state changes, the controller based on mathematical model needs to change the parameters affected in the model to achieve optimal performance.

Meanwhile, Reinforcement Learning (RL) has developed significantly in recent years which has been applied in many fields, from wireless Internet of Things, manufacturing to industrial process control [16-18]. In recent years, Deep Reinforcement Learning (DRL) has attracted wide attention in solving high-dimensional control with high complexity [19]. DDPG is one of the model-free DRL methods for continuous action spaces and has been widely applied in many fields, such as robotic control [20], manipulator control [21] and wireless sensors [22].

The stability of the control system with neural networks was proved by Lyapunov method in [23,24] and DRL for control has made great progress in recent years [25]. Many researchers applied DDPG to industrial process control which has characteristics of large disturbance and strong coupling and achieved a series of results [18]. In [26], the authors applied the DDPG method in heating, ventilation, and air conditioning(HAVC) to achieve continuous and accurate thermal control of the system. Ma et al. [27] applied DDPG to a polymerization system with strong non-linearity, large time delay, and noise tolerance. The DDPG controller learned the optimal strategy as the optimal control strategy for controlling nonlinear valves in [28]. Spielberg et al. [29] extended the DDPG to process control problems, and evaluated their approach on SISO Systems, Multi Input Multi Output(MIMO) systems and tested it under various scenarios. Notice that the DDPG method can operate without knowing mathematical models and increase stability against uncertainty in the thickness and tension control problem of unsteady rolling process.

Therefore, the main contributions are listed as follow:

1. In this paper, the model-free DDPG algorithm is applied in the thickness and tension control problem of unsteady rolling process with random disturbance and complex uncertainty for the first time, which overcomes the inaccurate modeling caused by random disturbance and coupling relationship of thickness and tension.

2. We map the thickness and tension control problem of unsteady rolling process to MDP by taking random disturbance in the unsteady rolling process into consideration, where action space of the agent and state of the environment were divided by state space model of steady-state rolling process and reward function was designed to overcome the absence of the mathematical model of unsteady rolling process and improve the control performance of unsteady rolling process.

3. Compared with the traditional PI controller, simulation results show that the DDPG controller has better accuracy, stability, and rapidity for the thickness tension in the unsteady rolling process. Compared with the maximum tension error of PI controller of $26.13 \%$, the maximum tension error of the DDPG controller is $11.58 \%$. The controller based on artificial intelligence improves the quality of the cold rolling strip and realizes intelligence.

The rest paper is organized as follows. In Section 2, we describe the unsteady rolling process and mapping thickness and tension control to Markov decision process. In Section 3, we set up the environmental network and apply the DDPG controller to solve the problem of thickness tension control in the unsteady rolling process. Section 4 provides training results and testing results which show that the DDPG controller has better stability and rapidity compared with a PI controller. Finally, some conclusions are shown in Section 5.

\section{Problem description}

\subsection{The unsteady rolling process}

The unsteady rolling process is a special part of the cold rolling process which includes acceleration, deceleration and over weld process, and so on. How to control the unsteady rolling process stably and accurately has become an urgent problem in the cold rolling process. The factors that make the unsteady rolling process hard to control are listed as follows:

Due to the non-linearity, time-varying in the unsteady rolling process coupled with various uncertainties such as variable lubrication state, variable equipment working condition, etc., it is difficult to establish an accurate mathematical model to describe the unsteady rolling process. Despite there is the steady-state mathematical model for reference, but the constant parameter mathematical model used in the actual control process is difficult to meet the requirements of continuous change 
which leads to low model matching. In addition, there are deviations in key parameters such as rolling force and forward slip in the actual production process which also leads to inaccurate results when using the existing model.
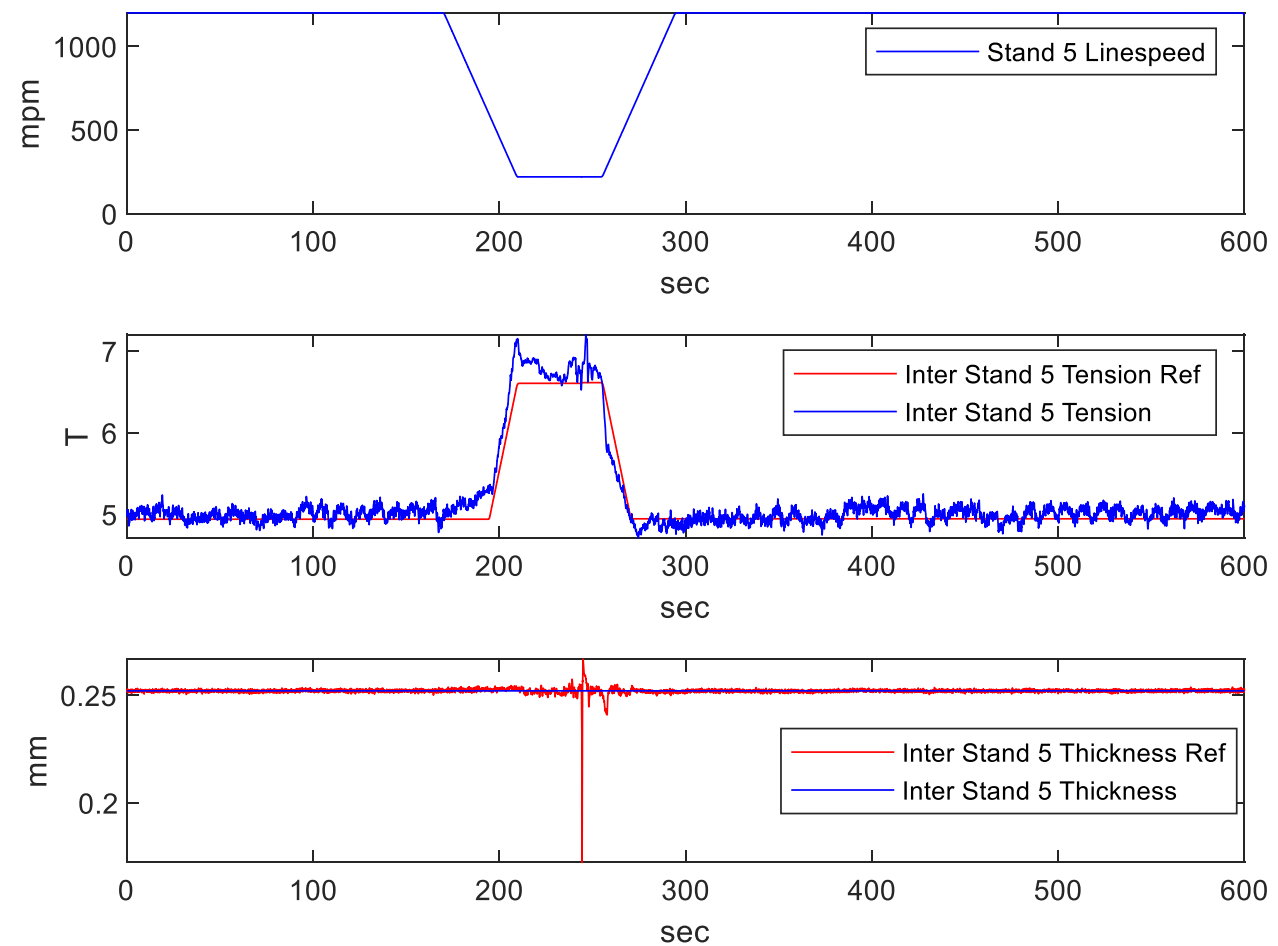

Fig. 2 Curve of thickness tension control in steady-state rolling process and the unsteady rolling process

Fig. 2 depicts the curve of thickness and tension control in the steady-state rolling process and the unsteady rolling process. It is noticed that the thickness control curve and tension control curve have good tracking performance to the target curve in the range of constant speed. However, in the range of velocity variation, the thickness control curve and tension control curve deviate from the target curve, which indicates that the control accuracy of thickness and tension in the acceleration and deceleration process with PI controller is insufficient.

In our work, we propose a controller based on DRL to alleviate the insufficient control accuracy of thickness and tension in the acceleration and deceleration process. In order to deal with the difficulty of accurately describing the unsteady rolling process by the mathematical model, we adopt the strategy of constructing a deep neural network to represent the mathematical model. The specific methods will be described in Section 3.

\subsection{State space model of thickness and tension}

According to the bounce equation of the rack, the incremental equation of the output thickness can be expressed as (1). The subscripts in , out , 0 and $i$ in formula respectively represent entry of stand, exit of stand, $i$ th stand and initial value.

$$
\Delta h_{\text {out }, i}=\Delta S_{i}+\frac{\Delta P_{i}}{M_{m}}
$$

where $h, S, P, M_{m}, h$ represent strip thickness, roll gap, rolling force, the mill stiffness respectively.

The rolling force is affected by input thickness, output thickness, front tension and back tension.

$$
\Delta P_{i}=\alpha_{1 i} \cdot \Delta h_{\text {in }, i}+\alpha_{2 i} \cdot \Delta h_{\text {out }, i}+\alpha_{3 i} \cdot \Delta T_{\text {in }, i}+\alpha_{4 i} \cdot \Delta T_{\text {out }, i}
$$

where $T$ is the tension of strip between adjacent stand, 
$\alpha_{1 i}=\frac{\partial P_{i}}{\partial h_{i n, i}}, \alpha_{2 i}=\frac{\partial P_{i}}{\partial h_{\text {out }, i}}, \alpha_{3 i}=\frac{\partial P_{i}}{\partial T_{\text {in }, i}}, \alpha_{4 i}=\frac{\partial P_{i}}{\partial T_{\text {out }, i}}$

According to (1) and (2), the formulation of output thickness increment model can be expressed as

$\Delta h_{\text {out }, i}=e_{1 i} \cdot \Delta S_{i}+e_{2 i} \cdot \Delta h_{\text {in }, i}+e_{3 i} \cdot \Delta T_{\text {in }, i}+e_{4 i} \cdot \Delta T_{\text {out }, i}$

where

$e_{1 i}=\frac{M_{m i}}{M_{m i}-\alpha_{2 i}}, e_{2 i}=\frac{\alpha_{1 i}}{M_{m i}-\alpha_{2 i}}, e_{3 i}=\frac{\alpha_{3 i}}{M_{m i}-\alpha_{2 i}}, e_{4 i}=\frac{\alpha_{4 i}}{M_{m i}-\alpha_{2 i}}$

In the rolling process, rolling mill exit forward slip $S_{h}$ and back slip $S_{H}$. The forward slip coefficient $f_{i}$ and the backward slip coefficient $b_{i}$ are defined as (6) and (7):

$$
\begin{gathered}
S_{h}=\frac{V_{\text {out }}-V}{V} \\
S_{H}=\frac{V-V_{\text {in }}}{V} \\
f_{i}=1+S_{h} \\
b_{i}=1-S_{H}
\end{gathered}
$$

where $V$ represents rolling speed.

According to the mass flow equation,

$$
V_{\text {in }} h_{\text {in }}=V_{\text {out }} h_{\text {out }}
$$

Backward slip coefficient is defined as,

$$
b_{i}=\frac{f_{i} \cdot h_{\text {out }, i}}{h_{\text {in }, i}}
$$

Forward slip is related to friction coefficient, deformation resistance and strip thickness and tension between stands, we defined the increment of forward slip coefficient as:

$\Delta f_{i}=\beta_{1 i} \cdot \Delta h_{\text {in }, i}+\beta_{2 i} \cdot \Delta h_{\text {out }, i}+\beta_{3 i} \cdot \Delta T_{i n, i}+\beta_{4 i} \cdot \Delta T_{\text {out }, i}$

where

$\beta_{1 i}=\frac{\partial f_{i}}{\partial h_{i n, i}}, \beta_{2 i}=\frac{\partial f_{i}}{\partial h_{\text {out }, i}}, \beta_{3 i}=\frac{\partial f_{i}}{\partial T_{i n, i}}, \beta_{4 i}=\frac{\partial f_{i}}{\partial T_{\text {out }, i}}$

The increment of rolling speed and backward slip coefficient can be expressed as:

$\Delta V_{i n, i}=\Delta V_{i} \cdot b_{i 0}+\Delta b_{i} \cdot V_{i 0}$

$$
\Delta V_{\text {out }, i-1}=\Delta V_{i-1} \cdot f_{i-1,0}+\Delta f_{i-1} \cdot V_{i-1,0}
$$

$\Delta b_{i}=\frac{\Delta f_{i} \cdot h_{\text {out }, i 0}}{h_{\text {in }, i 0}}+\frac{f_{i 0} \cdot \Delta h_{\text {out }, i}}{h_{\text {in }, i 0}}-\frac{f_{i 0} \cdot h_{\text {out } i 0}}{h_{\text {in }, i 0}^{2}} \Delta h_{\text {in }, i}$

According to Hooke's law and the tension formula between frames, the change rate of tension increment between frames can be written as:

$$
\frac{d\left(\Delta T_{i n, i}\right)}{d t}=\frac{E B h_{i n, i 0}}{L_{i}} \cdot\left(\Delta V_{\text {in }, i}-\Delta V_{\text {out }, i-1}\right)
$$

Combined with the above formula, the change rate of tension increment is deduced as follows, 


$$
\begin{aligned}
& \frac{d\left(\Delta T_{i n, i}\right)}{d t}=\frac{E B h_{i n, i 0}}{L} \cdot\left\{-V_{i-l, o} \cdot\left(\beta_{2 i-1} \cdot e_{3 i-1}+\beta_{3 i-1}\right) \cdot \Delta T_{i n, i-1}\right. \\
& +\left[\frac{V_{i o}}{h_{i n, i o}}\left(h_{\text {out }, i o} \cdot \beta_{2 i} \cdot e_{3 i}+h_{\text {out }, i o} \cdot \beta_{3 i}+f_{\text {io }} \cdot e_{3 i}\right)-V_{i-l, o} \cdot \beta_{2 i-1} \cdot e_{4 i-1}-V_{i-l, o} \cdot \beta_{4 i-1}\right] \cdot \Delta T_{i n, i} \\
& +\frac{V_{i o}}{h_{\text {in }, i o}}\left(h_{\text {out }, i o} \cdot \beta_{2 i} \cdot e_{4 i}+h_{\text {out }, i o} \cdot \beta_{4 i}+f_{\text {io }} \cdot e_{4 i}\right) \cdot \Delta T_{\text {in }, i+l}-V_{i-l, o} \cdot\left(\beta_{I i-I}+\beta_{2 i-1} \cdot e_{2 i-1}\right) \cdot \Delta h_{\text {in, }, i-1} \\
& +\frac{V_{\text {io }}}{h_{\text {in }, i o}}\left(h_{\text {out }, i o} \cdot \beta_{1 i}+h_{\text {out }, i o} \cdot \beta_{2 i} \cdot e_{2 i}+f_{i o} \cdot e_{2 i}-f_{i o} \cdot h_{\text {out }, i o} / h_{\text {in }, i o}\right) \cdot \Delta h_{\text {in }, i} \\
& \left.-f_{i-l, o} \cdot \Delta V_{i-I}+b_{i o} \cdot \Delta V_{i}-V_{i-l, o} \cdot \beta_{2 i-I} \cdot e_{I i-I} \cdot \Delta S_{i-l}+\frac{V_{i o} \cdot e_{I i}}{h_{i n, i o}} \cdot\left(h_{\text {out }, i o} \cdot \beta_{2 i}+f_{i o}\right) \cdot \Delta S_{i}\right\} \\
& =\xi_{1} \cdot \Delta T_{i n, i-1}+\xi_{2} \cdot \Delta T_{i n, i}+\xi_{3} \cdot \Delta T_{i n, i+1}+\xi_{4} \cdot \Delta h_{i n, i-1}+\xi_{5} \cdot \Delta h_{i n, i} \\
& +\xi_{6} \cdot \Delta V_{i-1}+\xi_{7} \cdot \Delta V_{i}+\xi_{8} \cdot \Delta S_{i-1}+\xi_{9} \cdot \Delta S_{i}
\end{aligned}
$$

where

$$
\begin{aligned}
& \xi_{l}=-\frac{E B h_{i n, i 0}}{L} \cdot V_{i-1, o} \cdot\left(\beta_{2 i-1} \cdot e_{3 i-1}+\beta_{3 i-1}\right) \\
& \xi_{2}=\frac{E B h_{\text {in }, i 0}}{L} \cdot\left[\frac{V_{\text {io }}}{h_{\text {in }, i o}}\left(h_{\text {out }, i o} \cdot \beta_{2 i} \cdot e_{3 i}+h_{\text {out }, i o} \cdot \beta_{3 i}+f_{i o} \cdot e_{3 i}\right)-V_{i-l, o} \cdot \beta_{2 i-l} \cdot e_{4 i-1}-V_{i-l, o} \cdot \beta_{4 i-1}\right] \\
& \xi_{3}=\frac{E B h_{i n, i o}}{L} \cdot \frac{V_{i o}}{h_{i n, i o}}\left(h_{\text {out }, i o} \cdot \beta_{2 i} \cdot e_{4 i}+h_{\text {out }, i o} \cdot \beta_{4 i}+f_{\text {io }} \cdot e_{4 i}\right) \\
& \xi_{4}=-\frac{E B h_{i n, i 0}}{L} \cdot V_{i-1, o} \cdot\left(\beta_{1 i-1}+\beta_{2 i-1} \cdot e_{2 i-1}\right) \\
& \xi_{5}=\frac{E B h_{i n, i o}}{L} \cdot \frac{V_{i o}}{h_{\text {in }, i o}}\left(h_{\text {out }, i o} \cdot \beta_{1 i}+h_{\text {out }, i o} \cdot \beta_{2 i} \cdot e_{2 i}+f_{i o} \cdot e_{2 i}-f_{\text {io }} \cdot h_{\text {out }, i o} / h_{\text {in }, i o}\right) \\
& \xi_{6}=-\frac{E B h_{i n, i 0}}{L} \cdot f_{i-1, o} \\
& \xi_{7}=\frac{E B h_{i n, i 0}}{L} \cdot b_{i o} \\
& \xi_{8}=-\frac{E B h_{i n, i 0}}{L} \cdot V_{i-1, o} \cdot \beta_{2 i-1} \cdot e_{1 i-1} \\
& \xi_{9}=\frac{E B h_{i n, i o}}{L} \cdot \frac{V_{i o} \cdot e_{1 i}}{h_{i n, i o}} \cdot\left(h_{\text {out }, i o} \cdot \beta_{2 i}+f_{i o}\right)
\end{aligned}
$$

Based on the field data and rolling experience, the parameter change rate of the actuator can be defined as,

$$
\begin{aligned}
& \frac{d \Delta S_{i}}{d t}=\frac{\Delta U_{S i}}{\tau_{S}}-\frac{\Delta S_{i}}{\tau_{S}}, S_{i}(0)=0 \\
& \frac{d \Delta V_{i}}{d t}=\frac{\Delta U_{V i}}{\tau_{V}}-\frac{\Delta V_{i}}{\tau_{V}}, V_{i}(0)=0
\end{aligned}
$$

where $\Delta U_{S i}$ and $\Delta U_{V i}$ are the roll gap target value and roll speed target value of the stand $i$ respectively. $\tau_{S}$ is time constant of ASR system and $\tau_{\mathrm{v}}$ is time constant of HGC system. The control is by adjusting the control signal $U_{S i}$ and $U_{V i}$ so that the thickness of strip and the inter-stand tension will track the reference value.

The control structure of stand 5 is shown in Fig. 3. Based on the above formula and state space theory, the state space model of tension and thickness between adjacent stand can be defined as:

$$
\left\{\begin{array}{l}
\frac{d \boldsymbol{x}}{d t}=\tilde{\boldsymbol{A}}_{i} \boldsymbol{x}_{i}(t)+\tilde{\boldsymbol{B}}_{i} \boldsymbol{u}_{i}(t)+\tilde{\boldsymbol{W}}_{i 1} \boldsymbol{d}_{i}(t) \\
\boldsymbol{y}_{i}(t)=\tilde{\boldsymbol{C}}_{i} \boldsymbol{x}_{i}(t)+\tilde{\boldsymbol{W}}_{i 2} \boldsymbol{d}_{i}(t)
\end{array}\right.
$$

where

$$
\begin{aligned}
& \boldsymbol{x}_{i}(t)=\left[\begin{array}{lll}
\Delta \boldsymbol{T}_{i n, i} & \Delta \boldsymbol{S}_{i} \Delta \boldsymbol{V}_{i-1}
\end{array}\right]^{T}, \quad \boldsymbol{u}_{i}(t)=\left[\Delta \boldsymbol{U}_{S i} \Delta \boldsymbol{U}_{V i-1}\right]^{T}, \\
& \boldsymbol{y}_{i}(t)=\left[\Delta \boldsymbol{h}_{\text {out }, i} \Delta \boldsymbol{T}_{i n, i}\right]^{T} \text {, }
\end{aligned}
$$




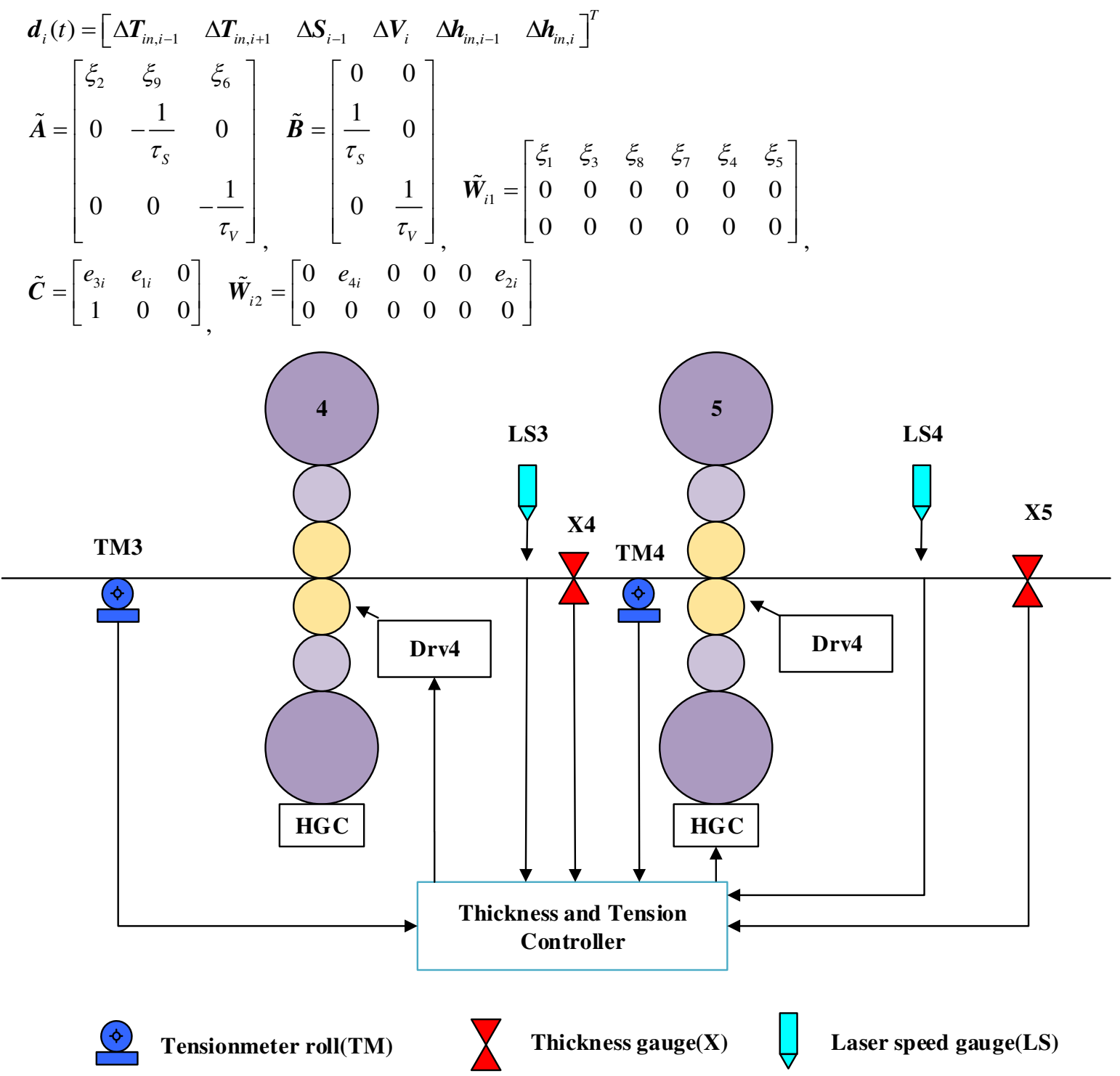

Fig. 3 Control structure of stand 5

\section{Optimal control with Deep Reinforcement Learning}

\subsection{RL and DDPG}

$\mathrm{RL}$ is an important branch of machine learning, RL algorithm maps the environment state detected by the agent to the action of the agent. Through a trial and error learning process in the environment, the agent takes the action of maximizing the long-term discount reward in (19) where the discount factor $0<\gamma<1$.

$$
R_{t}=\sum_{k=0}^{\infty} \gamma^{k} r_{t+k}=r_{t+1}+\gamma r_{t+2}+\gamma^{2} r_{t+3}+\cdots
$$

Many RL algorithms, such as Q-learning, SARSA, DQN, and so on, rely on the action-value function estimated from experience which is called value-based RL algorithm. Trial-and-error search and delayed rewards are prominent features of RL and provide a solid foundation for RL. In essence, the action-value function estimates the total reward $R_{t}$ from a state $s$ by taking a certain action follow policy $\pi$. The action-value function is expressed by (20) 


$$
Q^{\pi}=\mathrm{E}_{\pi}\left[\mathrm{R}_{t} \mid s, a\right]=\mathrm{E}_{\pi}\left[\sum_{k=0}^{\infty} \gamma^{k} r_{t+k} \mid s, a\right]
$$

Once the action-value function $Q^{\pi}$ converges to the optimal policy with sufficient experience, the action taken in each state will change the current state to the state with the highest action-value function estimate.

Usually, the action-value function is represented by nonlinear function approximators. If action-value functions are not generalized, the architecture of the action-value function will no longer be applicable [30]. DQN and DDPG updated the weights $\theta^{Q}$ of the Q-network iteratively with minimizing the loss function $L$.

$$
L=\frac{1}{N} \sum_{i}\left(\mathrm{y}_{i}-Q\left(s_{i}, a_{i} \mid \theta^{Q}\right)^{2}\right)
$$

RL was initially proposed to deal with the discrete action-space problem, but there are many situations that require continuous action [31]. An algorithm called actor-critic based on policy gradient for continuous space is proposed by Sutton [32]. The actor-critic consists of two parts: one is the actor network updated by the policy gradient, the other is the critic network eatimating the action-value function $Q^{\pi}$.

Fig. 4 shows the schematic diagram of the actor-critic algorithm.

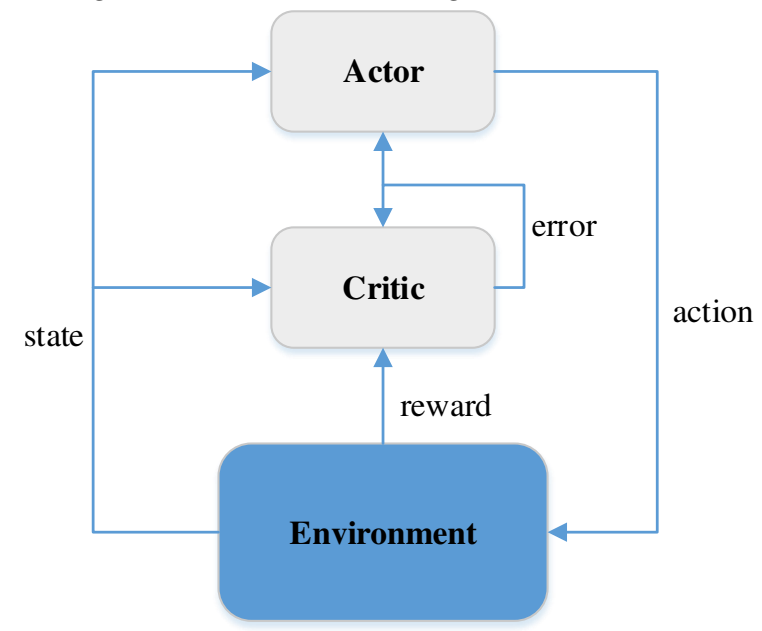

Fig. 4 Schematic diagram of actor-critic algorithm

However, the actor-critic algorithm needs a long training cycle, and it is difficult to converge to the optimal result. Lilllicrap et al. developed DDPG based on learning in mini-batch from DQN to ensure convergence and improve training speed [33]. Replay buffer is used in DQN to store historical samples $\left[s_{t}, a_{t}, r_{t}, s_{t+1}\right]$. In the training period, the network's weight is updated by the mini-batch tuple data that were sampled randomly.

Referring to the process from DQN to DDQN, DDPG also adopts the concepts of the target network and current network. The weight updating speed of the target network is slow, which greatly improves the stability of the network [34]. DDPG is a deterministic policy, different from the actor-critic algorithm, its actor policy is updated by the sampled gradient $\left.\nabla_{\theta^{\prime \prime}} \pi\right|_{s_{i}}$ :

$$
\left.\left.\left.\nabla_{\theta^{\mu}} \pi\right|_{s_{i}} \approx \frac{1}{N} \sum_{i} \nabla_{a} Q\left(s, a \mid \theta^{Q}\right)\right|_{s=s_{i}, a=\pi\left(s_{i}\right)} \nabla_{\theta^{\mu}} \pi\left(s \mid \theta^{\mu}\right)\right|_{s_{i}}
$$

The target network is slowly updated by (23) and (24).

$$
\begin{aligned}
\theta^{Q^{\prime}} & \leftarrow \tau \theta^{Q}+(1-\tau) \theta^{Q^{\prime}} \\
\theta^{\mu^{\prime}} & \leftarrow \tau \theta^{\mu}+(1-\tau) \theta^{\mu^{\prime}}
\end{aligned}
$$

\subsection{Mapping thickness and tension control to MDP}

In this subsection, we map the thickness and tension control problem of unsteady rolling process as a Markov Decision Process(MDP), which is then solved by a model-free DRL algorithm in Section 3.3.

MDP is a mathematical model of the sequential decision in RL [34]. It describes the completely 
observable environment, including four basic elements: state $s$, action $a$, reward $r$, state transition probability $p$. In MDP, the agent constantly interacts with its environment, chooses actions $a$ according to policy, and the environment responds to it by presenting a new situation $s_{t+1}$, and the environment also gives feedback reward $r$ to the agent.

According to the state space model of single stand in (18), the thickness and tension of strip steel at the current time are only affected by the previous state parameters and not by the state parameters at any other time.

In the thickness and tension control problem of unsteady rolling process, the four basic elements are described as follows:

State $s: 1)$ current entry tension of $i-1$ th stand $T_{i n, i-1}^{t}$;2) current entry tension of $i+1$ th stand $T_{i n, i+1}^{t}$;3) current entry tension of $i$ th stand $\left.T_{i n, i}^{t} ; 4\right)$ current roll gap of $i-1$ th stand $S_{i n, i-1}^{t} ; 5$ ) current roll gap of $i$ th stand $\left.S_{i n, i}^{t} ; 6\right)$ current rolling speed of $i$-1th stand $V_{i-1}^{t} ; 7$ )current rolling speed of $i$ th stand $\left.V_{i}^{t} ; 8\right)$ current entry thickness of $i$ th stand $h_{i n, i-1}^{t}$;9)current entry thickness of $i$ th stand $\left.h_{i n, i}^{t} ; 10\right)$ current exit thickness of $i$ th stand $h_{\text {out } i,}^{t}$;

Notice that the state parameters include disturbance term $\boldsymbol{d}_{i}(t)$, input term $\boldsymbol{x}_{i}(t)$ and output term $\boldsymbol{y}_{i}(t)$ of state space model.

Action $a: 1)$ The set-point of roll gap $\left.U_{S i}^{t} ; 2\right)$ The set-point of Roll speed $U_{V i-1}^{t}$

Action includes control term $\boldsymbol{u}_{i}(t)$ of state space model which $U_{S i}^{t}$ and $U_{V i-1}^{t}$ are continuous variable.

We define tension error $\Delta T_{i n, i}^{t}$ and thickness error $\Delta h_{\text {out }, i}^{t}$ as follows:

$$
\begin{aligned}
\Delta T_{i n, i}^{t} & =\mathrm{T}_{i n, i}^{t}-\mathrm{T}_{\text {ref }} \\
\Delta h_{\text {out }, i}^{t} & =\mathrm{h}_{\text {out }, i}^{t}-h_{\text {ref }}
\end{aligned}
$$

Reward: We design the reward function by comprehensively considering the accuracy of thickness and tension of rolling stand and the convergence efficiency of the agent training process, which is defined as follows:

$$
r=\left\{\begin{array}{l}
-\left(\Delta T_{i n, i}^{t}{ }^{2}+\Delta h_{\text {out }, i}^{t}{ }^{2}\right)+0.08, \text { if }\left(\Delta T_{i n, i}^{t}{ }^{2}+\Delta h_{\text {out }, i}^{t}{ }^{2}\right) \leq 0.1 \\
-\left(\Delta T_{i n, i}^{t}{ }^{2}+\Delta h_{\text {out }, i}{ }^{2}\right)+0.05, \text { if } 0.1 \leq\left(\Delta T_{i n, i}^{t}{ }^{2}+\Delta h_{\text {out }, i}^{t}{ }^{2}\right) \leq 0.2 \\
-\left(\Delta T_{i n, i}^{t}{ }^{2}+\Delta h_{\text {out }, i}^{t}{ }^{2}\right), \text { else }
\end{array}\right.
$$

We define the reward function as the negative number of the square sum of tension error and thickness error. In order to accelerate the convergence speed of agent training, we take the method of giving additional rewards to agents. When the error is between $10 \%$ and $20 \%$, the agent will be given an additional reward of 0.05 . When the error is less than $10 \%$, the agent will be given an additional reward of 0.08 . The MDP of the thickness and tension control problem of unsteady rolling process is illustrated in Fig. 5.

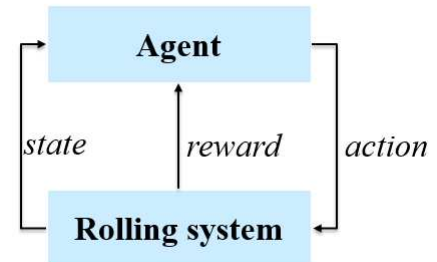

Fig. 5 MDP of the thickness and tension control problem of unsteady rolling process

For the above MDP, the state transition probability $p$ is not defined. The state transition probability is the probability distribution of state transition to other states after agents take certain actions $U_{S i}^{t}$ and $U_{V i-1}^{t}$ in a certain state. When the state transition probability is known, the MDP is completely observable, the cumulative reward can be solved by some iterative methods. In the thickness and tension control problem of unsteady rolling process, obtaining accurate state transition probability models is a complex task, because an accurate mathematical model is difficult to establish as mentioned in Section 2.

Based on the above considerations, we adopted the model-free DDPG algorithm to overcome the 
uncertainties of the unsteady rolling process and cross-coupling between stands. Without prior knowledge of the environment or state transition, the model-free DDPG algorithm learns the control strategy of thickness tension from the environment with the growth of the training epoch by interacting with the environment.

\subsection{Thickness and tension control framework}

In the thickness and tension control problem of unsteady rolling process, the simulation starts from a random initial state. In each step of each episode, the action $a_{t}$ is generated by the deterministic policy of the actor network with random OU noise for exploration [31]. The noise decreases with the increase of epochs. The neural network simulating the rolling state will return a set of training data to the replay buffer at each time step, in which the reward value is calculated according to the formula in (26).

After storing enough experience in the replay buffer, the optimal strategy is learned by random sampling in small batches. The update of the critic online network is updated by (21), and the action network is updated by (22). After each training step, the target critic network and the target action network are slowly updated by (23) and (24).

The specific process is shown in Fig. 6:

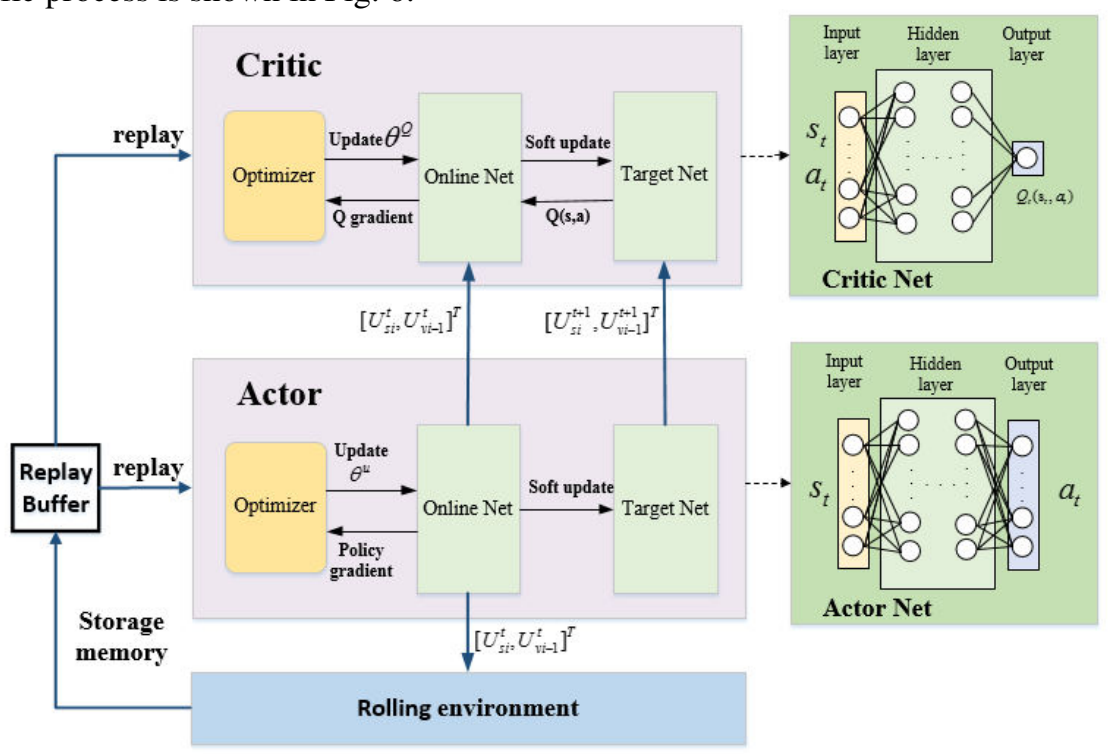

Fig. 6 The framework of thickness and tension control

The pseudocode of DDPG algorithm for thickness and tension control in cold rolling is included as follows:

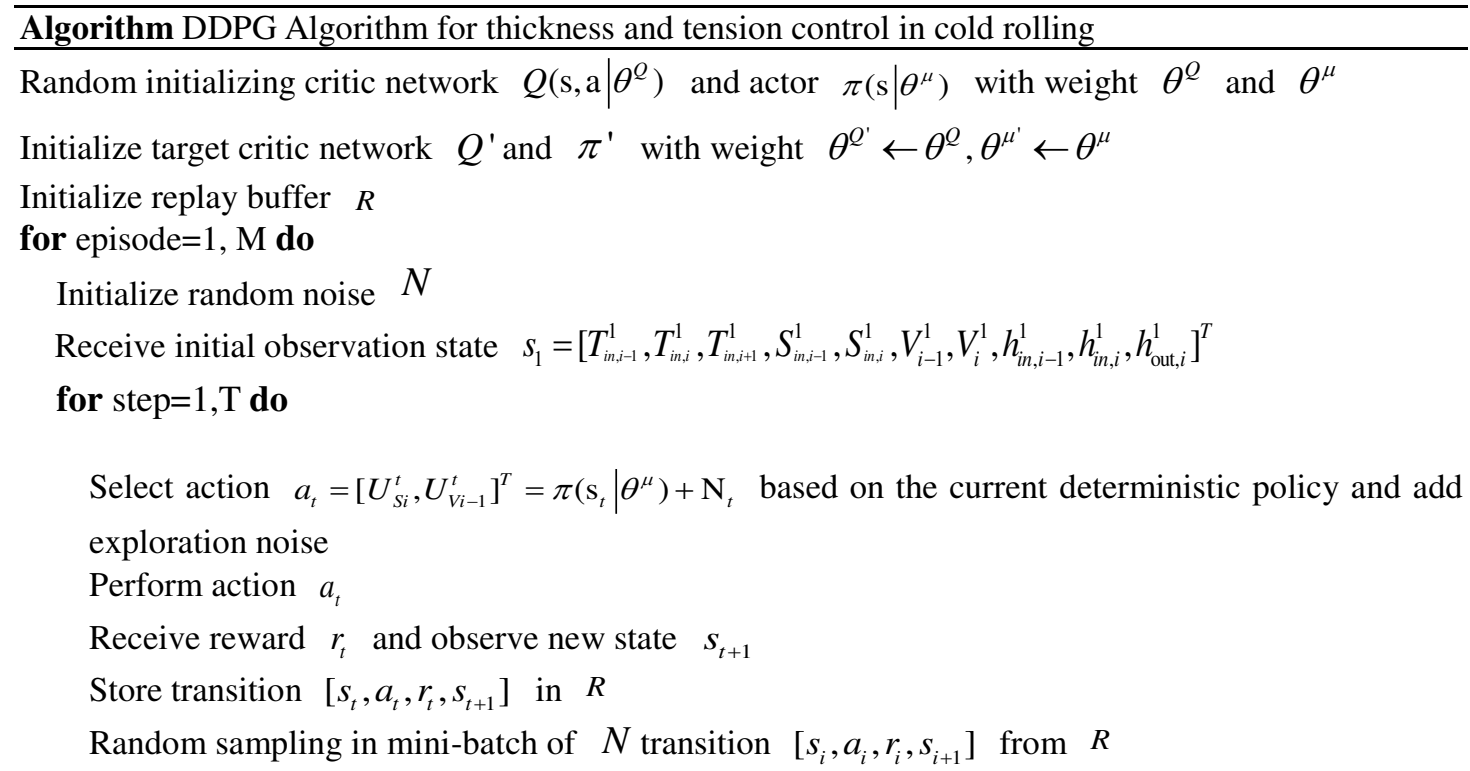


Set $y_{i}=r_{i}+\gamma Q^{\prime}\left(\mathrm{s}_{i+1}, \pi^{\prime}\left(\mathrm{s}_{i+1} \mid \theta^{\mu^{\prime}}\right) \mid \theta^{Q^{\prime}}\right)$

Update critic by minimizing the loss: $L=\frac{1}{N} \sum_{i}\left(\mathrm{y}_{i}-Q\left(s_{i}, a_{i} \mid \theta^{Q}\right)^{2}\right)$

Update the actor policy using the sampled gradient:

$$
\left.\left.\left.\nabla_{\theta^{\mu}} \pi\right|_{s_{i}} \approx \frac{1}{N} \sum_{i} \nabla_{a} Q\left(s, a \mid \theta^{Q}\right)\right|_{s=s_{i}, a=\pi\left(s_{i}\right)} \nabla_{\theta^{\mu}} \pi\left(s \mid \theta^{\mu}\right)\right|_{s_{i}}
$$

Update the target networks:

$$
\begin{aligned}
\theta^{Q^{\prime}} & \leftarrow \tau \theta^{Q}+(1-\tau) \theta^{Q^{\prime}} \\
\theta^{\mu^{\prime}} & \leftarrow \tau \theta^{\mu}+(1-\tau) \theta^{\mu^{\prime}}
\end{aligned}
$$

end for

end for

The advantage of the DDPG controller is that it can carry out continuous control, and improves the training efficiency and stability compared with the controller based on actor-critic algorithm [31]. The DDPG controller has become one of the ideal candidates in the industrial process control field.

\section{Simulation result}

The training and test results of thickness and tension control of the DDPG controller of unsteady rolling process are given in this section. We compare the control effect in thickness and tension control of the PI controller and the DDPG controller to further illustrate the superiority of the DDPG controller. The results show that the DDPG controller has better stability and rapidity.

\subsection{Simulation environment based on depth neural network}

In this subsection, we define the simulation environment of the thickness and tension control problem of unsteady rolling process.

According to the theory of MDP mentioned in the above Section, the simulation environment should accept the current state and action to present a new situation of next time interval and reward. We use a data-driven neural network to simulate this process, and train the neural network as the simulation environment with real data. The action and the current state are the input vectors of the environment network, and the state at the next time interval is defined as the output of the environment network to simulate the rolling stand in the thickness and tension control problem of unsteady rolling process. Considering the faster training speed and better convergence, different types of output data and input data of the environment network are normalized to $[0,1]$.

Considering the complexity of the thickness and tension control problem of unsteady rolling process, an environment network with seven fully connected layers is used in the simulation environment, which has 512 and 256 nodes in the third and fourth hidden layer, respectively. And to normalize the output data to $[0,1]$, we adopt sigmoid as the activation function of the output layer. In the thickness and tension control problem of unsteady rolling process, the output thickness of stand 5 directly affects the product quality, so this paper simulates the control of stand 5 in the deceleration process.

In order to better evaluate the performance of the environment network, $10 \%$ of the deceleration process data are extracted as the test set. Fig. 7 shows the convergence trend of accuracy over epoch. The accuracy of the environment network reaches 0.8 after 200 epochs and 0.9 after 700 epochs. At last, the training accuracy was maintained at 0.93 and stabilized. 


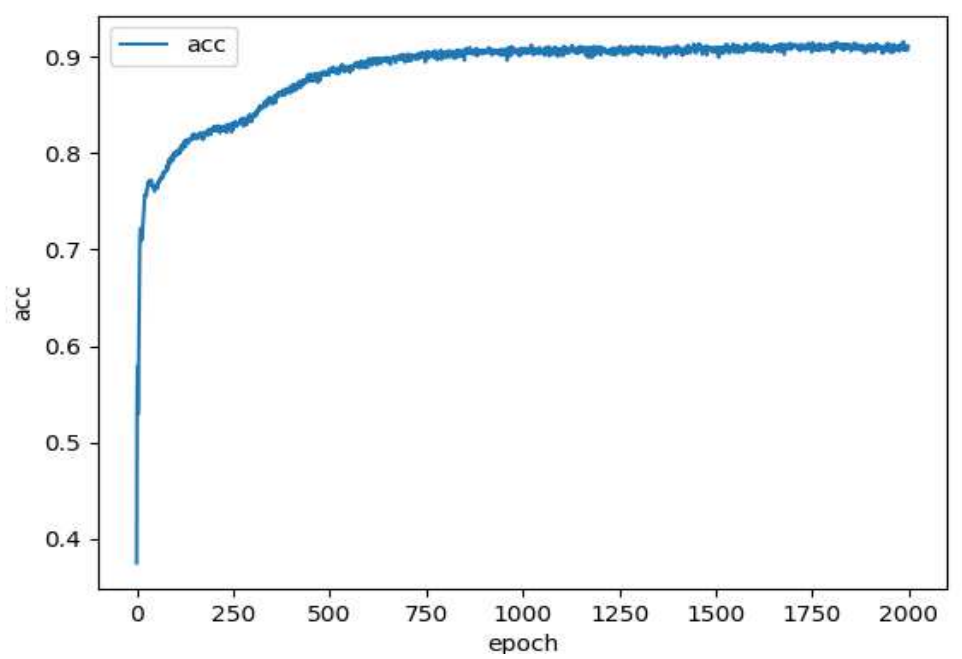

Fig. 7 The convergence trend of accuracy over epoch

The accuracy of the trained environment network is in the test set $92.44 \%$, indicating that the environment network has good generalization and can well characterize the unsteady rolling process of the rolling mill in the deceleration process.

\subsection{Training result}

We construct a framework of thickness tension control in the unsteady rolling process on Python platform based on tensorflow [35] package. We set the action network as five fully connected layers with $32,64,256,128$, and 32 neurons respectively. Similarly, we set the critic network as five fully connected layers, and the number of neurons is $64,256,128,16$, and 1 respectively. For 3000 epochs of training, it takes about 11 hours to complete in Intel Core i5-4200h CPU.

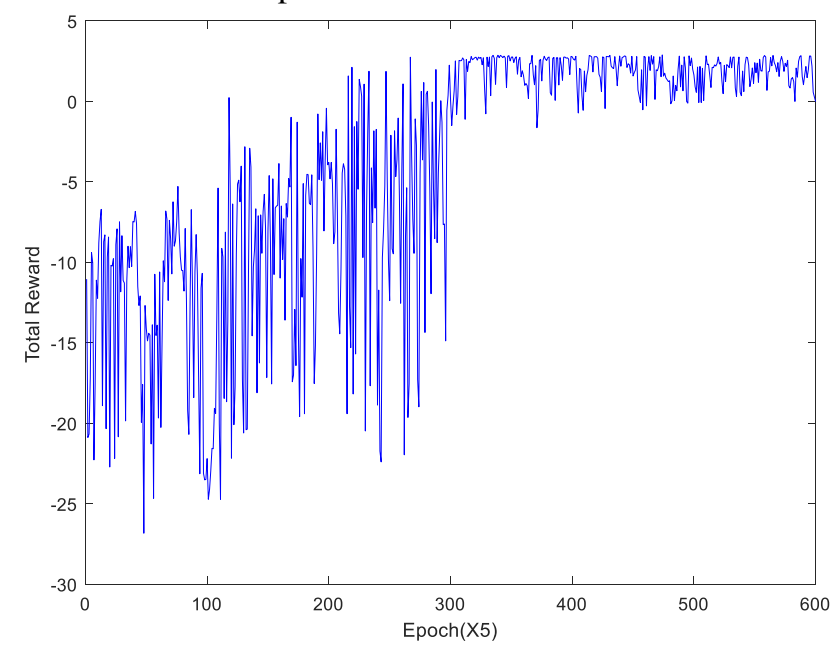

Fig. 8 Total reward of each epoch vs. training epochs

We set the time interval of each epoch to $5 \mathrm{~s}$, each step to $0.1 \mathrm{~s}$, and each epoch contains 50 steps. Fig. 8 shows the total reward of each epoch vs. training epochs. In order to better reflect the convergence, we sampled every five epochs of 3000 epochs. The total reward converges in about 1600 epochs, and it takes about 5 hours for the DDPG controller to learn the optimal control strategy.

\subsection{Test result}

In this subsection, we compare the control performance in thickness and tension control of the PI controller and the DDPG controller to further illustrate the superiority of the DDPG controller. Fig. 9 (a) shows the curve of rolling speed of the deceleration process in the unsteady rolling process. The results in Fig. 9(b)(c) show that the actions the DDPG controller takes are quite different from PI controller in deceleration process, in which the PI controller takes a smooth control action. The resulting trajectories of the DDPG controller follow closely to the target curve in the thickness and tension control problem 
of unsteady rolling process in Fig. 9(d)(e), but the curve of PI controller fluctuates up and down and does not track the target curve well. The strategy adopted by the DDPG controller is to increase the exit thickness of stand 5 and reduce the entry tension of stand 5 to resist the subsequent random disturbance.

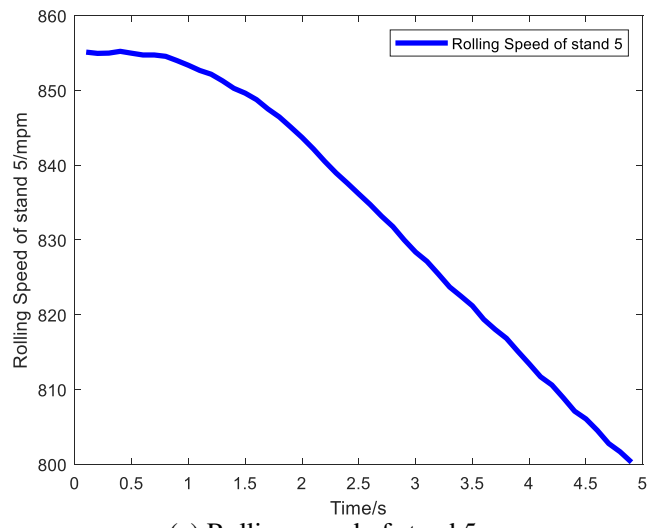

(a) Rolling speed of stand 5

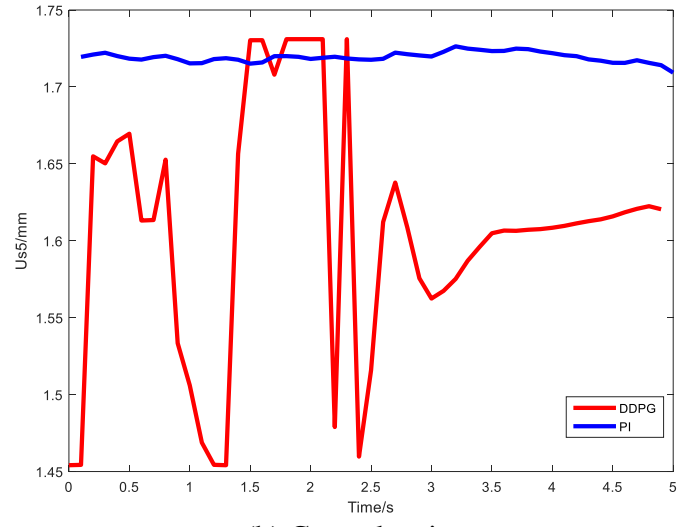

(b) Control action $U_{\mathrm{ss}}$

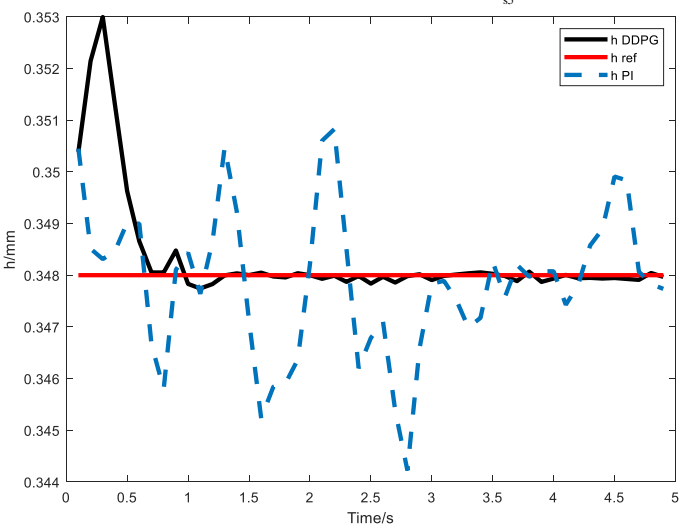

(d) Output thickness of stand 5

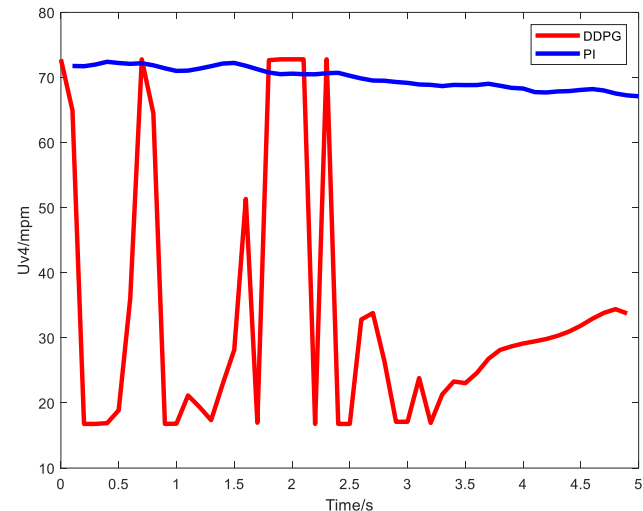

(c) Control action $U_{\mathrm{v} 4}$

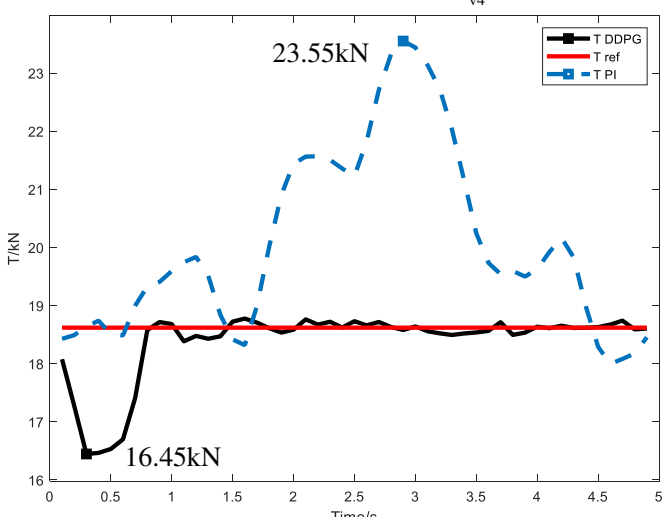

(e) Input tension of stand 5

Fig. 9 Control performance comparison between DDPG controller and PI controller in the decelerated rolling process

From the comparison of control results in Figure. 9(d)(e), the maximum tension error of the DDPG controller is $11.65 \%$ compared with $26.48 \%$ to PI controller which the set-point of tension is $18.62 \mathrm{kN}$. In dealing with the unsteady rolling process with random disturbance, DDPG controller has better stability and accuracy than the traditional PI controller, and the effect of thickness and tension control are well improved. Notice that the DDPG controller learns the control strategy without any prior knowledge to resist the random disturbance in the control system by using the strategy of sampling the replay buffer in mini-batch and using target network and current network to update the actor network.

\section{Conclusion}

This paper develops a the DDPG controller for the thickness and tension control problem of 
unsteady rolling process. The controller realizes the coupling control of thickness and tension in the deceleration process. By comparing, the DDPG controller has better control performance for thickness and tension in the unsteady rolling process than PI controller. DDPG improves control accuracy and stability of the process by maximizing the value of the long-term reward function. We also prove that the DDPG controller has strong adaptability in complex process control fields with random disturbance, strong coupling and nonlinearity. In addition, because the DDPG algorithm is a model-free RL algorithm, the control performance of the controller based on the DDPG algorithm will not be affected by the change of rolling environment. In summary, the controller based on the DDPG algorithm can control thickness and tension of the unsteady rolling process without prior knowledge. DRL can be used to solve complex industrial problems and is different from other control methods, which indicates that the controller based on the DRL method has a great application prospect in industrial process control.

In future work, we will use parallel actors in a distributed system to comprehensively control the whole cold rolling thickness tension control system. We may also consider the prioritized replay mechanism to improve the training efficiency in the next research.

Author contribution Wenying Zeng: conceptualization, methodology, investigation, data curation, software, formal analysis, experiment, and writing of the manuscript. Jinkuan Wang: conceptualization, resources, funding acquisition, supervision, project administration, and review. Yan Zhang: data collection and curation, writing review and editing. Yinghua Han: methodology, supervision, and writing including review and editing. Qiang Zhao: methodology, supervision, and writing including review and editing.

Funding This work was supported by National Natural Science Foundation of China (U1908213), Colleges and Universities in Hebei Province Science Research Program (QN2020504).

Data availability The data are available on reasonable demand.

Code availability The code is available on reasonable demand.

\section{Declarations}

Ethics approval All authors understand and approve the ethical responsibilities of the authors.

Consent to participate The authors consent to participate.

Consent for publication The authors consent to transfer the copyright of the article to publish.

Conflict of interest All authors declare that they have no conflict of interest.

\section{Reference}

[1] Zhang, X., Zhang, Q. , \& Sun, C. . (2009). Gauge And Tension Control In Unsteady State Of Cold Rolling Using Mixed $\mathrm{H} 2 / \mathrm{H} \infty$ Control. In: IEEE International Conference on Control and Automation Christchurch, New Zealand, pp 9-11

[2] Seung-Ho, S., \& Seung-Ki , S. A new tension controller for continuous strip processing line. IEEE Transactions on Industry Applications, 36, 2. doi:10.1109/28.833782

[3] Wang, Y., Xia, J., Wang, Z., \& Shen, H. (2020). Design of a fault-tolerant output-feedback controller for thickness control in cold rolling mills. Applied Mathematics and Computation, 369. doi:10.1016/j.amc.2019.124841

[4] Friebel, T., Zabet, K., Haber, R., \& Jelali, M., (2015) Predictive functional control of tandem cold metal rolling. In: 2015 IEEE Conference on Control Applications. pp 324-329

[5] Li, B., Fan, X. , Jiang, C., \& Jiang, G. (2014). Decoupling control of thickness and tension based on DRNN-PID in cold-rolling. In: Proceeding of the 11th World Congress on Intelligent Control and Automation, pp 1180-1184 
[6] Tan, S., Wang, L., Liu, J., (2014). Research on Decoupling Method of Thickness and Tension Control in Rolling Process. In: Proceeding of the 11th World Congress on Intelligent Control and Automation Shenyang, pp. 4715-4717

[7] Shiqi, A., (2016). The Decoupling Control of Tandem Cold Rolling Tension and Gauge. In: 2016 3rd International Conference on Information Science and Control Engineering (ICISCE), pp. $1154-1158$

[8] Hu, Y.-J., Sun, J., Wang, Q.-L., Yin, F.-C., \& Zhang, D.-H. (2018). Characteristic analysis and optimal control of the thickness and tension system on tandem cold rolling. The International Journal of Advanced Manufacturing Technology. doi:10.1007/s00170-018-3088-1

[9] Hu, Y., Sun, J., Chen, S. Z., Zhang, X., Peng, W., \& Zhang, D. (2019). Optimal control of tension and thickness for tandem cold rolling process based on receding horizon control. Ironmaking \& Steelmaking, 1-11. doi:10.1080/03019233.2019.1615813

[10] Koofigar, H. R., Sheikholeslam, F., \& Hosseinnia, S. (2011). Unified gauge-tension control in cold rolling mills: A robust regulation technique. International Journal of Precision Engineering and Manufacturing, 12(3), 393-403. doi:10.1007/s12541-011-0051-6

[11] Ogasahara, T., Hovd, M., Asano, K., (2016). Explicit Model Predictive Controller Design for Thickness and Tension Control in a Cold Rolling Mill. IFAC-PapersOnLine, 49(20), 126-131

[12] Hu, Y., Sun, J., Peng, W., \& Zhang, D. (2021). Nash equilibrium-based distributed predictive control strategy for thickness and tension control on tandem cold rolling system. Journal of Process Control, 97, 92-102. doi:10.1016/j.jprocont.2020.11.014

[13] Ozaki, K., Ohtsuka, T., Fujimoto, K., Kitamura, A., \& Nakayama, M. (2010). Nonlinear Receding Horizon Control of Thickness and Tension in a Tandem Cold Mill with a Variable Rolling Speed. Tetsu-to-Hagane, 96(7), 459-467. doi:10.2355/tetsutohagane.96.459

[14] Cao, L., Li, X., Wang, Q., \& Zhang, D. (2021). Vibration analysis and numerical simulation of rolling interface during cold rolling with unsteady lubrication. Tribology International, 153. doi:10.1016/j.triboint.2020.106604

[15] Sun, B., He, M., Wang, Y., Gui, W., Yang, C., \& Zhu, Q. (2018). A data-driven optimal control approach for solution purification process. Journal of Process Control, 68, 171-185. doi:10.1016/j.jprocont.2018.06.005

[16] Frikha, M. S., Gammar, S. M., Lahmadi, A., \& Andrey, L. (2021). Reinforcement and deep reinforcement learning for wireless Internet of Things: A survey. Computer Communications, 178, 98-113. doi:10.1016/j.comcom.2021.07.014

[17] Viharos, Z. J., \& Jakab, R. (2021). Reinforcement Learning for Statistical Process Control in Manufacturing. Measurement, 182. doi:10.1016/j.measurement.2021.109616

[18] Nian, R., Liu, J., \& Huang, B. (2020). A review On reinforcement learning: Introduction and applications in industrial process control. Computers \& Chemical Engineering, 139. doi:10.1016/j.compchemeng.2020.106886

[19] Du, Y., Zandi, H., Kotevska, O., Kurte, K., Munk, J., Amasyali, K., et al. (2021). Intelligent multi-zone residential HVAC control strategy based on deep reinforcement learning. Applied Energy, 281. doi:10.1016/j.apenergy.2020.11611

[20] Gu, S., Ethan, H., Timothy, L., Sergey, L., (2017) Deep reinforcement learning for robotic manipulation with asynchronous off-policy updates. In: Proceedings of the 2017 IEEE International Conference on Robotics and Automation (ICRA), pp 3389-3396

[21] Liu L., Chen E., Gao Z., Wang Y. (2019) Research on Motion Planning of Seven Degree of Freedom Manipulator Based on DDPG. In: Wang K., Wang Y., Strandhagen J., Yu T. (eds) Advanced Manufacturing and Automation VIII. IWAMA 2018. Lecture Notes in Electrical Engineering, vol 484. Springer, Singapore. https://doi.org/10.1007/978-981-13-2375-1_44

[22] Qiu, C., Hu, Y., Chen, Y., \& Zeng, B. (2019). Deep Deterministic Policy Gradient (DDPG)-Based Energy Harvesting Wireless Communications. IEEE Internet of Things Journal, 6(5), 8577-8588. doi:10.1109/jiot.2019.2921159

[23] Wang, Y., Shen, H., \& Duan, D. (2017). On Stabilization of Quantized Sampled-Data Neural-Network-Based Control Systems. IEEE Transactions on Cybernetics, 47(10), 3124-3135. doi:10.1109/tcyb.2016.2581220

[24] Qi, Z., Peng, S., Honghai, L., \& Shengyuan, X. (2012). Neural-Network-Based Decentralized Adaptive Output-Feedback Control for Large-Scale Stochastic Nonlinear Systems. IEEE Transactions on Systems, Man, and Cybernetics, Part B (Cybernetics), 42(6), 1608-1619. doi:10.1109/tsmcb.2012.2196432

[25] Buşoniu, L., de Bruin, T., Tolić, D., Kober, J., \& Palunko, I. (2018). Reinforcement learning for control: Performance, stability, and deep approximators. Annual Reviews in Control, 46, 8-28. doi:10.1016/j.arcontrol.2018.09.005 
[26] Gao, G., Li, J., \& Wen, Y. (2020). DeepComfort: Energy-Efficient Thermal Comfort Control in Buildings Via Reinforcement Learning. IEEE Internet of Things Journal, 7(9), 8472-8484. doi:10.1109/jiot.2020.2992117

[27] Ma, Y., Zhu, W., Benton, M. G., \& Romagnoli, J. (2019). Continuous control of a polymerization system with deep reinforcement learning. Journal of Process Control, 75, 40-47. doi:10.1016/j.jprocont.2018.11.004

[28] Siraskar, R. (2021). Reinforcement learning for control of valves. Machine Learning with Applications, 4. doi:10.1016/j.mlwa.2021.100030

[29] Spielberg, S., Gopaluni, R, B., Loewen, P, D., (2017) Deep reinforcement learning approaches for process control. In: 2017 6th International Symposium on Advanced Control of Industrial Processes(AdCONIP), pp: 28-31

[30] Mnih, V., Kavukcuoglu, K., Silver, D., Graves, A., Antonoglou, L., \& Wierstra, D., et al. (2013) Playing Atari with Deep Reinforcement Learning. arXiv:1312.5602

[31] Lillicrap, T, P., Hunt, J, J., Pritzel, A., Heess, N., Erez, Y., \& Tassa, Y., et al. (2015) Continuous Control with Deep Reinforcement Learning. arXiv:1509.02971

[32] Sutton, R, S., McAllester, D., Singh, S., Mansour, Y., (2000) Policy gradient methods for reinforcement learning with function approximation. In: Advances in Neural Information Processing Systems, pp. 1057-1063

[33] Mnih, V., Kavukcuoglu, K., Silver, D., Rusu, A. A., Veness, J., Bellemare, M. G., et al. (2015). Human-level control through deep reinforcement learning. Nature, 518(7540), 529-533. doi:10.1038/nature14236

[34] Fang, X., Han, Y., Wang, J., \& Zhao, Q. (2019). A cognitive control approach for microgrid performance optimization in unstable wireless communication. Neurocomputing, 355, 168-182. doi:10.1016/j.neucom.2019.04.048.

[35] Abadi, M., Barham, P., Chen, J., Chen, Z., Davis, J., et al. (2016) Tensorflow: a system for large-scale machine learning. OSDI, 16, 265-283 